\title{
Atmospheric Cluster Dynamics Code: a flexible method for solution of the birth-death equations
}

\author{
M. J. McGrath ${ }^{1,2}$, T. Olenius ${ }^{1}$, I. K. Ortega ${ }^{1}$, V. Loukonen ${ }^{1}$, P. Paasonen ${ }^{1}$, T. Kurtén ${ }^{1}$, M. Kulmala ${ }^{1}$, and \\ H. Vehkamäki ${ }^{1}$ \\ ${ }^{1}$ Department of Physics, P.O. Box 64, University of Helsinki, 00014, Helsinki, Finland \\ ${ }^{2}$ Department of Biophysics, Graduate School of Science, Kyoto University, Kyoto 606-8502, Japan
}

Correspondence to: M. J. McGrath (mcgrath@ theory.biophys.kyoto-u.ac.jp)

Received: 23 August 2011 - Published in Atmos. Chem. Phys. Discuss.: 9 September 2011

Revised: 6 December 2011 - Accepted: 21 February 2012 - Published: 2 March 2012

\begin{abstract}
The Atmospheric Cluster Dynamics Code (ACDC) is presented and explored. This program was created to study the first steps of atmospheric new particle formation by examining the formation of molecular clusters from atmospherically relevant molecules. The program models the cluster kinetics by explicit solution of the birth-death equations, using an efficient computer script for their generation and the MATLAB ode15s routine for their solution. Through the use of evaporation rate coefficients derived from formation free energies calculated by quantum chemical methods for clusters containing dimethylamine or ammonia and sulphuric acid, we have explored the effect of changing various parameters at atmospherically relevant monomer concentrations. We have included in our model clusters with $0-4$ base molecules and $0-4$ sulfuric acid molecules for which we have commensurable quantum chemical data. The tests demonstrate that large effects can be seen for even small changes in different parameters, due to the non-linearity of the system. In particular, changing the temperature had a significant impact on the steady-state concentrations of all clusters, while the boundary effects (allowing clusters to grow to sizes beyond the largest cluster that the code keeps track of, or forbidding such processes), coagulation sink terms, non-monomer collisions, sticking probabilities and monomer concentrations did not show as large effects under the conditions studied. Removal of coagulation sink terms prevented the system from reaching the steady state when all the initial cluster concentrations were set to the default value of $1 \mathrm{~m}^{-3}$, which is probably an effect caused by studying only relatively small cluster sizes.
\end{abstract}

\section{Introduction}

Atmospheric aerosol particles are known to have significant effects on both the global climate and human health (Pöschl, 2005). Secondary aerosols form as a result of a series of events, starting with the clustering of individual molecules and progressing through growth into stable particles (Kulmala, 2003). Atmospheric particle formation has been observed to occur across a wide range of climates and environments (Kulmala et al., 2004), but probing the initial steps (where clusters measure one nanometer across or less) is still difficult, despite recent advances in instruments to measure both ionic and neutral clusters of very small size (Hirsikko et al., 2011; Vanhanen et al., 2011; Sipilä et al., 2009, 2010; Petäjä et al., 2011). Experimental and theoretical studies indicate that atmospheric particle formation involves sulfuric acid and water (see, for example, Brus et al. (2011) and references therein), but these two components are not enough to explain all the observed particle formation events. Possible candidates to enhance sulfuric acid-water based particle formation are ammonia and dimethylamine (Kurtén et al., 2008).

Theory provides a useful tool to explore the nanometersized molecular clusters that are difficult to study experimentally. This paper gives an account of a method to solve the birth-death equations (BDE, the equations which describe the creation and destruction of molecular clusters by condensation and evaporation) as related to atmospheric clusters. Courtney (1962) explicitly solved the BDE equations for clusters up to one hundred molecules of water. Nishioka and Fujita (1994) looked at the binary water/sulfuric acid 
system, solving the equations by using Euler's method. Wyslouzil and Wilemski (1995) examined six different systems using the Bulirsch-Stoer method. Vehkamäki et al. (1994) looked at two binary systems and solved the concentrations for the steady state using a matrix method. McGraw (1995) did something similar for the water-acid system. All of the previous studies made an assumption that only monomers can collide and evaporate from the clusters, except for McGraw (1995), who reasoned that the acid hydrate is the most stable cluster (and therefore will be present in the highest concentration), so collisions with the hydrate are more important than with the bare acid monomer.

Improvements to the assumptions made in the above studies have also been carried out. For example, Arstila (1997) studied the effect of non-monomer collisions/evaporations for pure water, acetic acid, and a combined water/sulfuric acid system. Kathmann et al. (2004) solved the equations for the steady-state rate using a matrix method for the binary water/ammonia system. Several studies have explored particle formation through nucleation in a confined system, i.e. where the monomer concentration is depleted during the course of the event (Kožíšek et al., 2004; Kožíšek and Demo, 2005; Kožíšsek et al., 2006). Efforts have also been made to solve the BDE for various systems using approximate methods instead of explicit solution of the system of differential equations (see, for example, Rao and McMurry (1989); Girshick and Chiu (1990); Koutzenogii et al. (1996); Chesnokov and Krasnoperov (2007) and references therein), in order to reduce the total number of equations to something manageable. In addition, some researchers have transformed the water/sulfuric acid and water/acid/ammonia system to a quasi-unary system, which also has the effect of reducing the total number of equations; these equations were then solved explicitly (Yu, 2005, 2006; Kazil and Lovejoy, 2007). Finally, Kulmala (2010) introduced the Dynamical Atmospheric Cluster Model for explicit solution of the BDE. In spirit, this model is closest to the work reported here, although the procedure for generating the modeling equations is quite different.

This manuscript reports the methodology behind a model for the prediction of particle formation rates and cluster concentrations in vapors. The procedure was developed for the exploration of atmospherically relevant systems, although extension to cluster formation events in other kinds of vapours is straightforward and demonstrated below. The methods presented here differ from previous efforts in two main ways: (1) all of the equations are generated via a computer script, allowing for greater flexibility and speed while reducing the possibility of typographical errors, and (2) the free energies for the evaporation coefficients can be taken from any source, including quantum mechanical calculations. The following section describes in detail how various parameters in the model were selected, separating the discussion into those parameters needed for electrically neutral systems and those involving charged molecules (e.g., ion-induced nucleation). Section 3 gives the results of various tests performed with the model, exploring the effect of changing the parameters/methods used on the steady-state concentrations and particle formation fluxes. Section 4 summarizes these tests and presents some concluding remarks.

\section{Methods}

The Atmospheric Cluster Dynamics Code (ACDC) is a dynamical model to study the time development of molecular cluster distributions by explicit solution of the birth-death equations. The birth-death equations can be written as

$$
\begin{aligned}
& \frac{d c_{i}}{d t}=\frac{1}{2} \sum_{j<i} \beta_{j,(i-j)} c_{j} c_{(i-j)}+\sum_{j} \gamma_{(i+j) \rightarrow i} c_{i+j}-\sum_{j} \beta_{i, j} c_{i} c_{j} \\
& -\frac{1}{2} \sum_{j<i} \gamma_{i \rightarrow j} c_{i}+Q_{i}-S_{i}
\end{aligned}
$$

where $i$ is the cluster whose concentration is given by this equation, $j$ is another cluster in the system, $c_{i}$ is the number density of cluster $i, \beta_{i j}$ is the collision coefficient between clusters $i$ and $j, \gamma_{i \rightarrow j}$ is the evaporation coefficient of a cluster $i$ into two smaller clusters (one of which is $j$ ), $Q_{i}$ is an outside source term of $i$, and $S_{i}$ includes other possible loss mechanisms for cluster $i$. The terms on the right hand side can be described physically as the generation (birth) of clusters of type $i$ through collisions of smaller clusters, the generation of clusters of type $i$ through evaporation of larger clusters, the destruction (death) of clusters of type $i$ through collisions with other clusters, the destruction of cluster $i$ by fragmentation into smaller clusters, other creation mechanisms, and other destruction mechanisms, respectively. These other mechanisms depend on the system being studied. We shall enumerate various such terms here for both neutral systems and those containing ions. All of the terms in the neutral system are also present in the ionic case, although the reverse is not true.

In ACDC, the BDEs are solved with the ode15s solver in MATLAB program, which is effective in solving systems of stiff differential equations (Shampine and Reichelt, 1997). As ACDC is solving the birth-death equations explicitly, the concentrations of all constituents are known as a function of time. In the interest of keeping the amount of material presented in this article concise, here we report only the steadystate results. The time-dependent data are, however, readily available and will be used as required by future applications. The novelty of ACDC is the generation of the equations that are fed into the MATLAB solver. Generation of the equations is essentially a series of logical checks over all possible cluster combinations to see which evaporations and collisions can create/destroy a given cluster. This work is tedious and prone to typographical errors if done by a human, but it is ideally suited to a computer code. In this case, 
we have chosen to use the Perl scripting language to generate the equations, due to the relative ease by which Perl handles string manipulation. This code was originally written to study the atmospherically-relevant system of sulfuric acid, ammonia, and bisulfate ion, but it has been designed to be flexible and can easily be extended to different systems (see Sect. 2.3 and Vehkamäki et al. (2012) for other examples). The equations can be modified through both an input file (which specifies the number and composition of clusters) and command-line arguments (which are used to add and remove various source/sink terms in the equations themselves).

It should be noted here that Eq. (1) contains all possible evaporations and collisions in the system. Many previous studies have limited themselves to the case of only monomer collisions and evaporations. This is a valid assumption in many cases where the monomer concentrations are much higher than those of other clusters, and it greatly lowers the complexity of the resulting equations. However, given that the generation of the equations in ACDC is done by a Perl script, there was only a small amount of programming effort required to include these terms, and it requires no special effort for the user. The existence of small stable clusters in the atmosphere also suggests that non-monomer collisions may be important (Vehkamäki et al., 2012). In addition, non-monomer evaporations might also become significant as the cluster size grows, as breaking into small stable clusters can be more energetically favorable than the evaporation of a monomer (Ortega et al., 2012). In any event, like most of the features in ACDC, non-monomer collisions and evaporations can be included or ignored by a simple command-line argument.

\subsection{Neutral clusters}

The collision coefficients between clusters are taken from the kinetic gas theory, and they describe how often two spherical clusters following a Maxwell-Boltzmann velocity distribution collide with each other. For clusters $i$ and $j$ the collision coefficient, $\beta_{i j}$ in $\mathrm{m}^{3} \mathrm{~s}^{-1}$ is given as

$\beta_{i j}=\left(\frac{3}{4 \pi}\right)^{1 / 6}\left(\frac{6 k_{b} T}{m_{i}}+\frac{6 k_{b} T}{m_{j}}\right)^{1 / 2}\left(V_{i}^{1 / 3}+V_{j}^{1 / 3}\right)^{2}$

where $k_{b}$ is the Boltzmann constant, $T$ is the temperature, and $m_{i}$ and $V_{i}$ are the mass and volume of cluster $i$, respectively. In ACDC, the volume of a cluster is determined in a simple fashion by assuming that the compounds in the cluster are in liquid form. The molecular volume of the pure liquid is calculated from the molecular mass and saturated liquid density (at an atmospherically relevant temperature), which are input parameters. The volume of the cluster is taken to be the sum of the molecular volume multiplied by the number of molecules of a given type for all molecular kinds in the cluster. While this is only an approximation, using more sophisticated methods (such as using the density of a solution with the same composition as the given cluster, or taking into account the temperature dependence of the liquid density) does not result in large differences to the steady-state cluster concentrations. Given the extra complexity of including such functions for every possible cluster composition, the difficulty of doing this for charged clusters, and the relative insensitivity of the final result, ACDC retains the simplest approach to determining cluster volume.

According to the condition of detailed balance, the evaporation coefficients can be calculated from the collision coefficients and the free energies of formation of the mother and daughter clusters:

$\gamma_{(i+j) \rightarrow i}=\beta_{i j} \frac{c_{i}^{e} c_{j}^{e}}{c_{i+j}^{e}}=\beta_{i j} c_{\text {ref }} \exp \left\{\frac{\Delta G_{i+j}-\Delta G_{i}-\Delta G_{j}}{k_{b} T}\right\}$

where $i$ and $j$ are the daughter clusters, $\beta_{i j}$ is the collision coefficient between $i$ and $j, c_{i}^{e}$ is the equilibrium concentration of cluster $i, \Delta G_{i}$ is the free energy of formation of cluster $i$ from the constituent monomers (which implies that all monomer free energies are zero), and $c_{\text {ref }}$ is the monomer concentration of the reference vapor for which the free energies were calculated. From ACDC's point of view, the origin of the free energies is not relevant, i.e. they can be calculated by any method from the classical liquid drop model to quantum chemistry. ACDC currently allows for one to enter the enthalpy $(\Delta H)$ and entropy $(\Delta S)$ of formation from the monomer in place of the free energy $(\Delta G=\Delta H-T \Delta S)$; this allows for quick exploration of different temperatures (under the often-used assumption that $\Delta H$ and $\Delta S$ remain constant over the temperature range of interest).

Two major loss terms which need to be included in any realistic system are the loss of particles on the walls of the chamber and the loss of particles by collision with large aerosols not explicitly included in the simulation, i.e. coagulation. The former term is only applicable in the case of laboratory experiments, while the latter is important when trying to predict the concentrations under atmospheric conditions where there are usually high populations of large pre-existing aerosols. This work assumes atmospheric conditions, and consequently the only loss term included in the simulations is the loss by coagulation. This term is derived from experimental data measured in Hyytiälä, Finland (Dal Maso et al., 2008). We use a constant coagulation sink coefficient of $2.6 \times 10^{-3} \mathrm{~s}^{-1}$, which is the median condensation sink coefficient of sulfuric acid vapor on pre-existing aerosol particles. Using the parametrized formula from Kulmala et al. (2001) for a cluster size dependent coagulation sink coefficient did not have a significant effect on the steady-state cluster concentrations.

One question that plagues the users of any simulation package in a finite system is that of boundary conditions. Boundary issues can manifest themselves in a code such as ACDC quite readily when the size of the largest tracked clusters is relatively small. This is because the code allows collisions to form large clusters which are not explicitly tracked 
in the system. As soon as these clusters form, they are "lost" (the material cannot re-enter the system). In the case where all the clusters on the boundary are unlikely to evaporate to smaller sizes, this loss does not affect the system significantly. However, this is not guaranteed to be the case for small systems. ACDC deals with this uncertainty by including an optional command-line flag to disable all collisions that result in clusters larger than what are included in the system. This option represents the opposite extreme of keeping all material in the system, and consequently running the code for the same system with both options gives an estimation of the boundary effects.

In addition to the steady-state cluster concentrations, one would also like to compute the flux clusters between different sizes and, by extension, the particle formation rate. The formation rate in our study is defined as the flux of clusters outside the system; since these clusters are not allowed to re-enter, it is as if they have become stable particles (a valid assumption if the clusters on the boundaries are large enough to have negligible evaporation coefficients). However, in smaller systems, it is not clear that this should be the case. Indeed, as Ortega et al. (2012) point out, for systems with stable pre-critical clusters even clusters larger than the critical nucleus can rapidly decay through non-monomer evaporation. This issue is explored more fully in Section 3 for our particular system of interest. ACDC automatically tracks the particle formation rate, and an extra command-line flag can be passed to keep track of the fluxes between clusters and the formation rates of all clusters, which makes for easy analysis after the simulation has been run.

\subsection{Ionic clusters}

If one is interested in ion-induced particle formation, the situation becomes a little more complex compared to the neutral case. All of the above terms are still included in the system, but additional questions must now be addressed. These questions include how the ions are introduced to the system (ion source terms), how the ions disappear from the system (recombination with ions of opposite charge), and how ions collide with neutral aerosols. These terms are included here for completeness, despite that the focus of this manuscript is on the neutral clusters.

A simple way to introduce ions into the system is to add a constant source term to the equation describing the concentration of the ionic form of a monomer of interest, similar to the way that other compounds (such as sulphuric acid and ammonia) are introduced to the system. This results in all ions being added to the system as monomers, which can then grow by combination with neutral clusters. However, from an experimental/observational standpoint, this is not very realistic. Ionization in the atmosphere is caused by cosmic rays or radiation from radon decay (Bazilevskaya et al., 2008). The concentration of sulfuric acid is much lower than the concentration of other air molecules, and consequently it is unlikely that the ion sources will be ionization of the sulfuric acid monomer (this is made even more unlikely by the fact that atmospheric sulfuric acid is mainly present as hydrates or attached to a base (Kurtén et al., 2011)).

Instead of adding a source term to the ionic form of e.g. the sulfuric acid monomer, ACDC has the option to introduce a new equation to the system. This equation keeps track of the concentration of a generic negative ion (it currently has the mass and molecular volume of an oxygen molecule, although the precise mass may be heavier in the troposphere).

$\frac{d c_{\text {ion }}}{d t}=Q_{\text {ion }}-\alpha_{\text {rec }} c_{\text {ion }} c_{\text {ion }}-\sum_{j} \beta_{\text {ion }, j} c_{\text {ion }} c_{j}$

In Eq. (4), $Q_{\text {ion }}$ is the ionization rate of the air (oxygen molecules), $\alpha_{\text {rec }}$ is the recombination rate of positive and negative ions (taken to be the usual literature value of $1.6 \times$ $10^{-12} \mathrm{~m}^{-3} \mathrm{~s}^{-1}$ (Israël, 1970; Bates, 1982)), and the summation is over every neutral cluster in the system. In addition to this equation, every neutral cluster has a loss term and every ionic cluster has a source term corresponding to the third term on the right in Eq. (4), which represents attachment of ions to neutral molecules. All ionic clusters also have loss terms corresponding to the recombination term, while all neutral clusters have a similar source term (it is assumed for simplicity that recombination produces a single neutral cluster, different to the mother ionic cluster only by conversion of the bisulfate ion to a neutral sulfuric acid).

It is well-known that the collision rate coefficient between ionic and neutral clusters is higher than between two neutral clusters (Langevin, 1905). In sulphuric acid containing systems, this is due to the fact that the ion interacts strongly with the permanent dipole moment of the acid molecule, resulting in more attractive forces and a larger collision cross-section. Consequently, Eq. (2) needs to be multiplied by an enhancement factor in the case that one of the clusters contains an ion (if both of the clusters contain ions of the same polarity, electrostatic repulsion will prevent then from colliding, so such collisions are not allowed in ACDC). The exact form of the enhancement factor is not well known, and several formulae exist. In ACDC, three factors are currently possible. The first one is to simply multiply every ion-neutral collision (and, because of detailed balance, every evaporation of an ionic cluster) by a constant factor (taken to be equal to ten). The second factor is given by Hoppel and Frick (1986), and depends on the size of the ionic cluster (using the rational that the more solvated the ion, the less impact it should have, so the value should tend towards unity as the cluster increases). The final option is given by Lovejoy et al. (2004), and depends also on the nature of the colliding cluster. Similarly, in the current model the coagulation sink of ionic clusters is enhanced by a factor of two. In future work containing ionic clusters, this effect will be examined in more detail. 


\subsection{Validation}

As with any other computational method, ACDC must be validated against a known system before the results can be analyzed in detail. That is, it must be shown to give the same answer as other methods for the same problem, or the differences must be thoroughly explained. The ordinary differential equation solvers in MATLAB (in particular, the ode $15 \mathrm{~s}$ routine used here) have already been well-tested and found to be robust (Shampine and Reichelt, 1997); therefore, what remains to be validated here is the method of generating the birth-death equations, i.e. the Perl script. Validation of this script has been performed in three ways.

The first way is the most straightforward: visual examination of the resulting equations. If the expected terms appear error-free in their entirety, the code is obviously working properly. This type of validation is done regularly on one or more of the equations to ensure the accuracy of the code. It can be safely assumed that if several of the equations are correct, all of the equations are correct given the methodical nature of computer codes. While continual inspection of this nature is certainly useful, it cannot be relied up by itself to confirm the accuracy of the method. Consequently, two other tests have been employed.

The second way is by comparison to classical nucleation theory. The equations of classical nucleation theory (CNT) give expressions for the steady-state nucleation (particle formation) rate and cluster concentrations in multi-component systems, which is a valid assumption when solving the birthdeath equations and considering only monomer collisions and evaporations. ACDC has been tested against CNT for the case of homogeneous water vapor-liquid nucleation and found to give differences of far less than $1 \%$ (Vehkamäki et al., 2012).

A final test is the nucleation of pure aluminum at high temperatures. Li et al. (2007) have determined very accurate monomer addition free energies using Monte Carlo simulation of the equilibrium constants and several high level corrections for the aluminum dimer through the 60-mer. Girshick et al. (2009) explored the kinetics of this system by using these addition free energies and modified collision rate coefficients (Li and Truhlar, 2008) to predict the nucleation rate and steady-state concentrations of the vapor-liquid nucleation of aluminum. Even without using the modified collision rate coefficients (i.e., using Eq. (2) above), ACDC provides results in very close agreement with what is seen by Girshick et al. (2009) This is perhaps unsurprising, since Girshick et al. (2009) use CNT as a basis for their dynamics calculations, which ACDC has already been validated against through the second test in this section, but it illustrates the flexibility of ACDC; the change from test two to test three required minor changes to the input file and the cluster free energies.

\section{Results}

When running ACDC, the first choice that needs to be made is the source of the thermodynamic data used to calculate the evaporation coefficients by Eq. (3). We have decided to perform all calculations here on the neutral acid/base (where the base can be either dimethylamine (DMA) or ammonia) clusters explored by Ortega et al. (2012). We wanted to include in our model only clusters for which we have commensurable high level quantum chemical data, thus restricting ourselves for clusters with a maximum of four acid and four base molecules. The free energies of formation were taken direction from the supplementary information of Ortega et al. (2012), which computes them using quantum mechanical methods that reproduce high level results to within an average of about $1 \mathrm{kcal} \mathrm{mol}^{-1}$ while being inexpensive enough to allow their use on large clusters. In the future, we will be able to add larger clusters when quantum chemical data becomes available with increased computer power, or through the use of liquid drop model properties for larger clusters. It should be noted that Nadykto et al. (2011) have recently published results on a similar system, which could also be used for this test; however, Ortega et al. (2012) report results for larger clusters, which reduces the boundary effects. Kurtén (2011) has also pointed out that the exchange/correlation density functional used by Nadykto et al. (2011) can significantly underestimate the stability of DMA/sulfuric acid clusters.

Water is not included in the system, because sufficient quantum chemical data for clusters containing water, sulfuric acid, and a base are not yet available. While this omission means that we are not examining a true atmospheric system in this manuscript, this is no shortcoming of the ACDC model itself. Rather, the effort required to compute the cluster free energies of water/acid/base clusters using quantum mechanical methods is quite significant for the clusters sizes we are exploring here. In fact, we have run test calculations on the quaternary $\mathrm{H}_{2} \mathrm{SO}_{4} / \mathrm{NH}_{3} / \mathrm{DMA} / \mathrm{H}_{2} \mathrm{O}$ system, for both neutral and negatively charged molecules, using both quantum mechanical free energies and those derived from the liquid drop model. The qualitative behavior of the system was not altered by the inclusion of water, although further simulations need to be performed when the quantum mechanical results for water are available.

As noted above, in smaller systems the flux out of the system does not give the actual particle formation rate. Since the evaporation of clusters outside the simulation system is artificially prevented, the simulated rate is larger than the actual formation rate if the clusters leaving the simulated system are not large enough to be stable against evaporation. This happens because some collisions occur which result in clusters not in the system, but which should (at least partially) evaporate back into the system. For example, the collision of the DMA monomer (which is present in a high concentration) with a cluster containing four DMA molecules (and any number of sulfuric acid molecules) forms a cluster that 

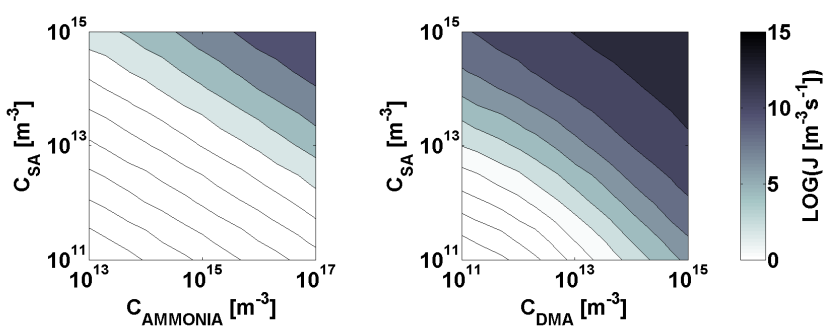

Fig. 1. The particle formation rate in sulfuric acid-ammonia (left panel) and sulfuric acid-dimethylamine (right panel) systems as a function of acid monomer and base monomer concentrations. The colour scale is shown on the right, and gives the base-10 logarithm of the particle formation rate defined in the text.

contains five amine molecules and is therefore not included in the system, but might not be very stable (Ortega et al. (2012) observed that clusters with more bases than acids are generally not stable). Nonetheless, this flux leaves the system and does not return. Because of this, we have decided to only allow clusters to leave the system by collisions where both colliding clusters contain sulfuric acid. All of the values of $J$ reported here were computed by:

$J=\sum_{i=1}^{4} \sum_{j=1}^{4} \sum_{k=0}^{4} \sum_{l=0}^{4} \beta_{i k, j l} \cdot c_{i k} \cdot c_{j l}$

where the indices $i$ and $j$ refer to the number of sulphuric acid molecules in the first and second cluster, and $k$ and $l$ refer to the number of base molecules, subject to the constraint that $i+j>4$ and/or $k+l>4$, so that the resulting cluster's concentration is not explicitly tracked in the simulation. It should be noted here that the largest clusters in our system are approximately $1 \mathrm{~nm}$ in diameter. As the particular formation rates given by Eq. (5) may be artificially overestimated, caution must therefore be stressed when comparing the rates presented here to true particle formation rates. In addition, the particle formation rate given by Eq. (5) is not the nucleation rate, and therefore care must be taken when comparing the values of $J$ reported here to true nucleation rates. In practice for our system of interest, the "steady state" means that the clusters in the system are in a steady state against formation from the gas phase, growth by condensation of gas phase molecules and cluster conditions, decay due to evaporation of molecules and cluster breakup, and coagulation onto pre-existing aerosols. The concentration of the clusters outside of the system is assumed to be zero.

Figure 1 shows the particle formation rate for sulfuric acidammonia and sulfuric acid-dimethylamine systems as a function of acid and base concentrations in $T=298.15 \mathrm{~K}$. The ranges of the sulfuric acid and ammonia monomer concentrations were chosen based on typical atmospheric concentrations measured in Hyytiälä (Janson et al., 2001; Petäjä et al., 2009). Because the monomer concentration in our system is not fixed, the source terms of each molecule type need to be set so they result in the desired steady-state monomer concentrations. This was done in the following way: first, the concentrations of the sulfuric acid and base monomers were set to the target constant values, i.e. the derivatives of the monomer concentrations were set to zero (Eq. 1). After the simulation finished, the source terms were calculated from the monomer equations in the steady state, and the simulation was re-run without fixing the monomer concentrations using these calculated source terms. Finally, it was checked that the monomer concentrations were within $3 \%$ of the desired values.

When examining the steady state properties of the system, one must always verify that the solutions to the system of differential equations have reached the steady state. The system was determined to be in the steady state when the concentrations of each component were within $0.1 \%$ of their concentrations at $50000 \mathrm{~s}$, as discussed in more detail below. All the simulations were initially run for $50000 \mathrm{~s}$ of simulation time, and the concentrations of species at several conditions were examined to ensure they reached the steady state. In addition, all of the rates in the right graph of Fig. 1 were run for $5000000 \mathrm{~s}$ (results not shown here); the rates differed only in the tenth significant figure or so, providing further evidence that $50000 \mathrm{~s}$ is enough to reach the steady state.

Several conclusions can be drawn from Fig. 1. The first is that the rate is higher in the DMA system than in the ammonia system at atmospherically relevant monomer concentrations. Therefore, we have chosen the sulfuric acid-DMA system to be our example system on which we will focus our attention. From Fig. 1 it can be seen that in the DMA system the rate depends more strongly on the sulfuric acid concentration than the DMA concentration, although there is certainly a dependence on the DMA concentration as well (e.g. raising the DMA concentration by an order of magnitude increases the rate by around three orders of magnitude at low acid/high DMA concentrations). At high acid concentrations, the DMA concentration does not have a large effect at least for the ranges studied here.

From Fig. 1, we can determine the conditions for the rest of the tests presented here. A typical atmospheric sulfuric acid concentration is $10^{12} \mathrm{~m}^{-3}$. If we take the DMA concentration to be equal to $5 \times 10^{13} \mathrm{~m}^{-3}$, ACDC predicts a particle formation rate of around $10^{6} \mathrm{~m}^{-3} \mathrm{~s}^{-1}$, which is a typical new particle formation rate seen in Hyytiälä (Dal Maso et al., 2005; Manninen et al., 2009). This DMA concentration is on the high range (but approximately the same order of magnitude) as what is measured in rural boreal areas (Ge et al., 2011). Therefore, we will use these concentrations as our "standard" concentrations for examining the effect of the other parameters below.

Figure 2 shows how long the concentrations in the system take to stabilize. This number is calculated by examining the concentrations throughout the entirety of the simulation and determining the smallest time at which the concentration of every species is within $0.1 \%$ of its final concentration. As 


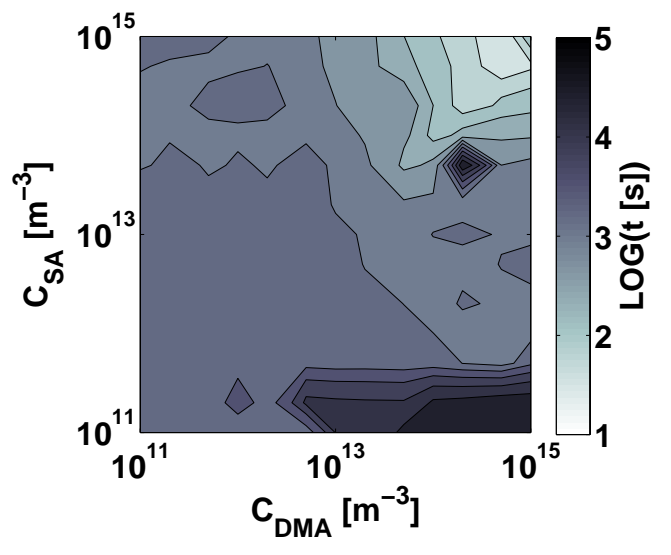

Fig. 2. The maximum length of simulation time required to converge the individual concentrations in the dimethylamine/sulfuric acid simulations, shown as a function of the monomer concentrations. The colour scale is shown on the right, and gives the base-10 logarithm of the time required for convergence (in seconds) as defined in the text.
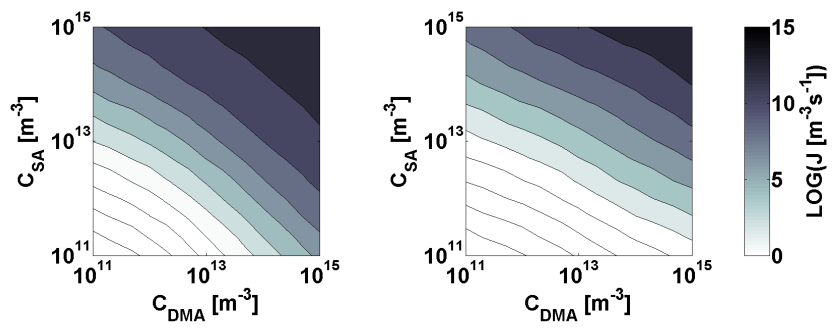

Fig. 3. The particle formation rate as a function of dimethylamine monomer and sulfuric acid monomer concentrations. The right plot considers only monomer evaporations and cluster collisions, while the left plot allows all evaporations and collisions. The colour scale is shown on the right, and gives the base-10 logarithm of the particle formation rate defined in the text.

this number is computed by looking at the final concentration and working backwards towards the beginning, there is no possibility that fluctuations in the concentrations greater than $0.1 \%$ occur after this time. From this graph, we can conclude several things. First of all is that all of the simulations do, in fact, reach the steady state within the $50000 \mathrm{~s}$ of simulation time. Secondly, the time required to reach the steady state is not affected much by the sulphuric acid concentration if the concentration of DMA is low, but becomes significant at higher base concentrations, with high concentrations of acid and base allowing the simulations to converge the quickest. It is interesting to note that the slowest times observed in Fig. 2 are on the order of several hours, which suggests that steadystate conditions are probably not observed in the atmosphere for this system.

Figure 3 shows the effect of only allowing monomer collisions and evaporations in the system. While this assumption (that non-monomer collisions and evaporations are not im-

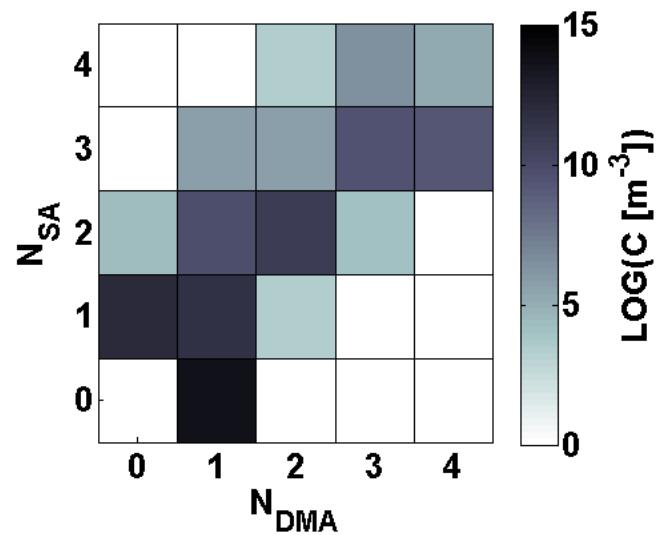

Fig. 4. The steady-state concentrations of clusters consisting of 0-4 sulfuric acid and 0-4 DMA molecules. The colour scale gives the base-10 logarithm of the concentration (in $\mathrm{m}^{-3}$ ).

portant) is valid under certain conditions (Arstila, 1997), it clearly won't be valid when there are stable pre-nucleation clusters (Vehkamäki et al., 2012). Stable pre-nucleation clusters correspond to a free energy minimum and have a negative formation free energy compared to (some) of the monomers, and thus their concentrations exceed the concentrations of (some of) the monomers. Therefore, the collision of any cluster with a stable pre-nucleation cluster is more likely than with a monomer. From Figure 3, we can see a rather large difference in the rates by excluding nonmonomer interactions (several orders of magnitude under certain conditions). This indicates that the concentrations of clusters in the system are comparable to those of the monomers, which suggests either local minima in the free energy surface at small clusters or at least relatively stable pre-critical clusters in this system; indeed, e.g. Ortega et al. (2012) have noticed the relatively stability of clusters consisting of two acids and two DMA molecules.

Figure 4 shows the steady-state concentrations of all the clusters when the monomer concentrations are set to the standard values described above. In Fig. 4, clusters with the highest concentrations are located around the diagonal of the acid and base number matrix. This seems sound, since clusters consisting mainly only of acid or base molecules are not expected to be stable (Ortega et al., 2012). Of particular interest is that the concentrations stay relatively high everywhere on the diagonal, i.e. larger clusters are also present in relatively high concentrations.

A plot of the total flux (collisions minus evaporations) is shown in Fig. 5. These pathways were determined by the following algorithm: (1) Every flux above a certain cutoff size $\left(10^{3} \mathrm{~m}^{-3} \mathrm{~s}^{-1}\right)$ was recorded, (2) For each flux, the path back to the monomer using the highest magnitude of flux for each step was traced. This means that not all flux pathways are shown, since there are numerous ways to get to most clusters from the monomers. For example, the concentration of one 


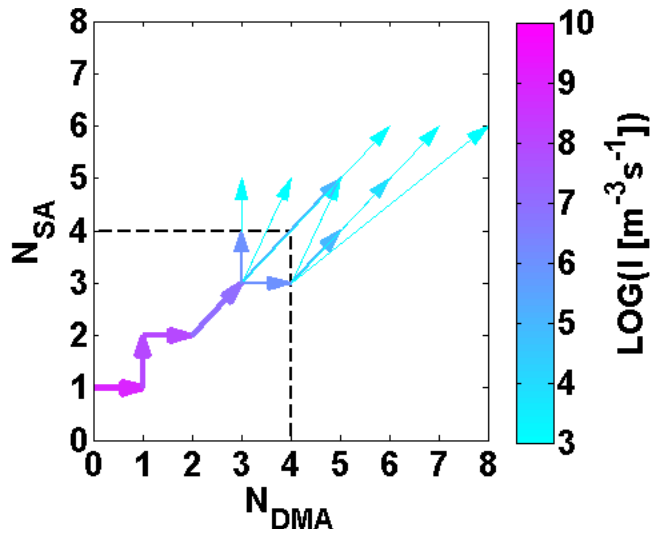

Fig. 5. The major movement of clusters out of the system. The system boundaries are depicted by dashed black lines. The line colour gives the total flux between clusters, and the arrow indicates the direction of the flux. Line thickness is used as an additional guide for the eye (thicker lines mean higher flux). The colour scale is the base-10 logarithm of the flux (given in $\mathrm{m}^{-3} \mathrm{~s}^{-1}$ ).
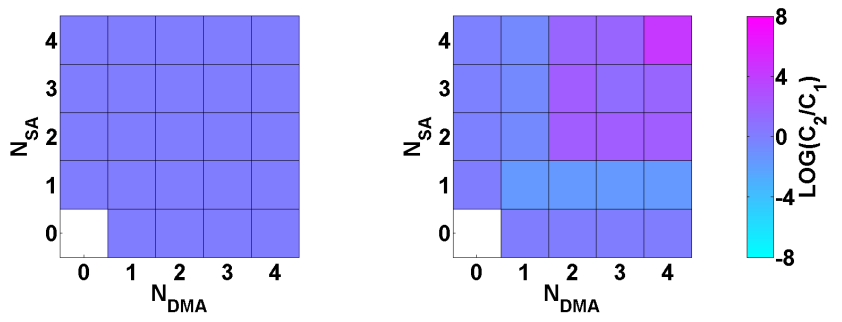

Fig. 6. The differences in cluster concentrations when the collisions resulting in clusters outside of the system are either (1) permitted or (2) prevented. The left panel is for the differences at the standard monomer concentrations, while the right panel is for high concentrations. The colour scale gives the base- 10 logarithm of the ratio of the concentrations in these two cases.

DMA/one acid clusters is high enough that self-collision to form the two amine/two acid cluster is significant; however, that collision is not included in Fig. 5 because the addition of one acid to form the two acid/one DMA cluster, followed by addition of a single DMA has a greater overall flux. It appears that the dominant pathway involves the formation of the one DMA/one acid cluster, which is very rapid (due to the high concentrations of the acid and DMA monomers). This cluster is stable enough to be present at a fairly high concentration, and could be a platform for growth into the larger sizes. It is interesting to note from this graph that the main flux out of the system is along the diagonal (equal numbers of acids and bases), and that significant fluxes are observed through non-monomer collisions.

The aforementioned boundary effects were examined with the option that disables collisions resulting in clusters outside of the system. Figure 6 shows the differences in concentrations with and without these collisions. It is important to

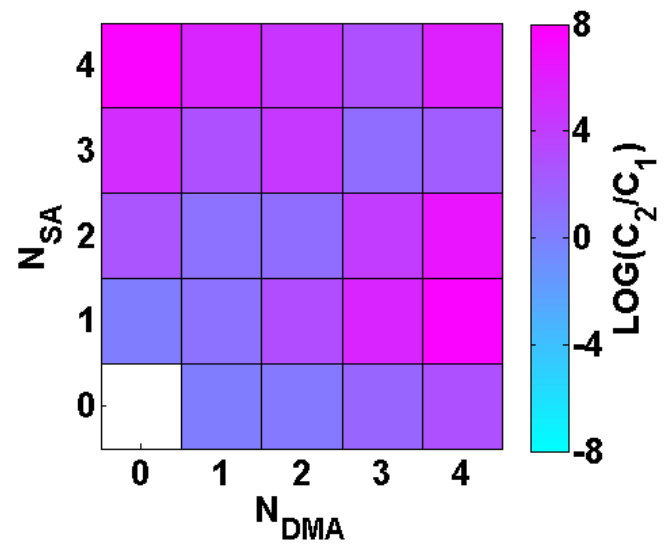

Fig. 7. The differences in cluster concentrations when the temperature is decreased from $298 \mathrm{~K}$ to $248 \mathrm{~K}$. The colour scale gives the base-10 logarithm of the ratio of the concentrations in these two cases.

make a brief note on the colouring scheme used in the next four figures here. Most importantly, the colour bar for all four figures is exactly the same. This enables a quick comparison between them, as strong colours on one plot means that the effect of changing that particular parameter is more significant than dull colours on a second plot. It should also be noted that any changes that are larger than this colour scale are now the same colour as the limit values, which attempts to prevent a single large change from skewing the whole colour scheme. A bit surprisingly, disallowing collisions from leaving the system did not have much effect on the concentrations in the chosen standard conditions (left panel of Fig. 6). This is probably due to the pathways out of the system by cluster collisions being much smaller in magnitude than those going to the coagulation sink or passing between clusters inside the system (which can somewhat be seen in Fig. 5). Therefore, this option was also tested at high monomer conditions (right panel, $\left[\mathrm{H}_{2} \mathrm{SO}_{4}\right]=10^{15} \mathrm{~m}^{-3}$ and $\left.\left[\mathrm{NH}\left(\mathrm{CH}_{3}\right)_{2}\right]=10^{15} \mathrm{~m}^{-3}\right)$, and this time the concentrations of large clusters increased as the clusters were kept inside the system. At higher concentrations, the flux out of the system from a certain cluster is now comparable to the flux to and from neighboring clusters, which means that eliminating this pathway results in a surplus of material to rearrange around the system. This, of course, results in higher cluster concentrations near the boundary.

An essential parameter in studying the dynamics of a molecular system is the temperature. Figure 7 shows the differences in concentrations when the temperature is decreased from $T_{1}=298 \mathrm{~K}$ to $T_{2}=248 \mathrm{~K}$. This range was chosen because it covers a broad range of tropospheric temperatures. As can be seen quite clearly in Fig. 7, decreasing the temperature increased the concentrations of almost all the non-monomer clusters. The increase in the concentrations can be explained by comparing the collision and 

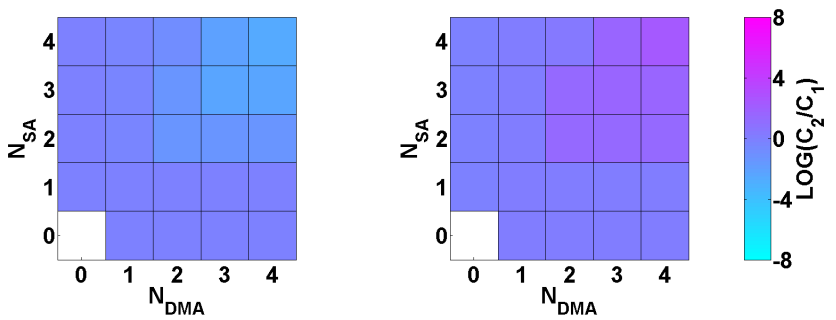

Fig. 8. The differences in cluster concentrations when the coagulation coefficient is increased by a factor of 10 (left panel) or turned off (right panel). The colour scale gives the base-10 logarithm of the ratio of the concentrations when compared to the standard conditions.

evaporation coefficients at both temperatures. As the temperature decreases, so do both the coefficients, but the decrease in the evaporation coefficients (where the temperature dependence is exponential) is much higher than that in the collision coefficients (where the dependence is in the square root of the temperature). Therefore, although the velocity of the particles decreases and they collide less frequently, they also stick together much more tightly, leading to more stable clusters. This is seen most dramatically in the clusters which are very unstable (the clusters which do not have around equal numbers of acids and bases).

One of the major particle sinks in the system is the condensation of clusters on large pre-existing aerosols (a similar effect to the condensation of clusters onto the chamber walls in laboratory experiments). The effects of increasing this sink or turning it off are shown in Fig. 8. It can be seen that the concentrations of the largest clusters are reduced by increasing the losses due to this sink. This is expected, since the flux will be attenuated with each step towards a larger cluster. It must be noted here that removing the coagulation sink terms resulted in the system not reaching the steady state if the initial monomer concentrations are set to the default value of $1 \mathrm{~m}^{-3}$. In this case, running for a simulation length of $5 \times 10^{4} \mathrm{~s}$ produced different cluster concentrations than running for $1 \times 10^{7} \mathrm{~s}$. However, when the initial monomer concentrations are set to the desired steady state values, the system does find a steady state, which is presented in Fig. 8 . In this case, the concentrations of the largest clusters are increased, which also is an expected result.

Since collisions between clusters play a major part in the birth-death equations, the question of sticking probabilities naturally arises. While many kinetic codes (including ACDC) increase the sticking probabilities for ion-neutral cluster collisions (as described above), to our knowledge the reverse case has not been explored in detail, i.e. when two neutral clusters collide, they will always stick together. However, there is no guarantee that this is always the case, and therefore the effect of sticking probabilities less than unity was examined. It has to be noted here that the sticking prob-

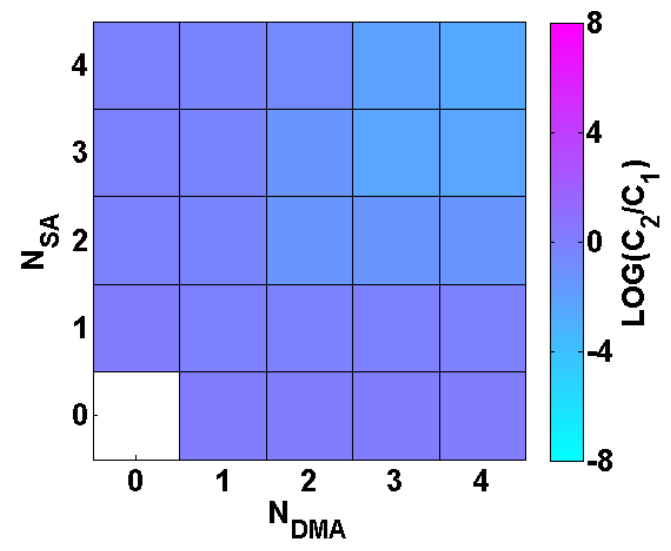

Fig. 9. The differences in cluster concentrations when the collision sticking probabilities of the most prevalent clusters in the system are reduced to 0.1 . The colour scale gives the base-10 logarithm of the ratio of the concentrations when compared to the standard conditions.

ability can also be thought to be taken into account in the evaporation (Kulmala and Wagner, 2001). Figure 9 shows the difference in concentrations when the sticking probability is reduced to 0.1 for collisions involving clusters that have the highest concentrations at the standard conditions. These clusters include the monomers and clusters consisting of (1) one acid and one DMA, (2) two acids and one DMA and (3) two acids and two DMA molecules. They were found to be the most important clusters regarding the effect of changing the sticking probability by performing the following test. First, the sticking probability in all collisions was set to 0.1 . Second, the sticking probabilities in collisions involving the aforementioned clusters were set to 0.1 , while probabilities in all the other collisions were reset to 1.0. These two tests gave the same results for the cluster concentrations. This implies that the sticking probabilities in collisions involving the most numerous clusters have the largest effect on the cluster distribution. As can be seen in Fig. 9, reducing the sticking probabilities has an effect on the largest clusters in the system, but it is not very strong. Reducing the sticking probabilities to 0.01 has a more significant effect (results not shown), but Kurtén et al. (2010) have shown that is is unlikely that the sticking factor would differ by that much from unity, especially for larger clusters.

\section{Conclusions}

We have presented a new program for modeling the kinetics of clusters by explicit solution of the birth-death equations, using an efficient computer script for their generation and the MATLAB ode15s routine for their solution. This script, referred to as the Atmospheric Cluster Dynamics Code (ACDC), can easily use cluster free energies calculated by any method, from liquid drop theory to quantum 
chemical calculations. By using the recent formation free energies computed by Ortega et al. (2012) for sulfuric acid and dimethylamine containing clusters, we have examined the effect of changing various system parameters at atmospherically relevant monomer concentrations, demonstrating that large effects can be seen for even small changes, due to the non-linearity of the system. Changing the temperature by $50 \mathrm{~K}$ has a large impact on the cluster distribution, while the boundary effects (i.e. allowing clusters to leave the system or keeping them inside the system boundaries), coagulation sink terms, non-monomer collisions, collision sticking coefficients, and monomer concentrations can all have significant effects under certain conditions. Removal of coagulation sink terms prevented the system from reaching the steady state when the initial monomer concentrations were set to $1 \mathrm{~m}^{-3}$, which probably results from the small system size. If the starting concentrations for monomers were set to the wanted steady state values, the system was able to find a steady state.

Acknowledgements. We gratefully acknowledge ERC StG 257360MOCAPAF, a National Science Foundation International Research Program grant (OISE-0853294, MJM), the Maj and Tor Nessling Foundation (project \#2010212, PP, project \#2011200, VL), and the Academy of Finland (Center of Excellence program project \#1118615, LASTU program project \#135054, project \#1127372) for funding. We would also like to thank Malcolm and Angus Young, Brian Johnson, Phil Rudd and Cliff Williams for inspiration.

Edited by: K. Lehtinen

\section{References}

Arstila, H.: Kinetic effect of cluster-cluster processes on homogeneous nucleation rates in one- and two-component systems, J. Chem. Phys., 107, 3196-3203, 1997.

Bates, D. R.: Recombination of small ions in the troposphere and lower stratosphere, Planet. Space Sci., 30, 1275-1282, 1982.

Bazilevskaya, G. A., Usoskin, I. G., Flückiger, E., Harrison, R. G., Desorgher, L., Bütikofer, R., Krainev, M. B., Makhmutov, V. S., Stozhkov, Y. I., Svirzhevskaya, A. K., Svirzhevsky, N., and Kovaltsov, G.: Cosmic ray induced ion production in the atmosphere, Space Sci. Rev., 137, 149-173, 2008.

Brus, D., Neitola, K., Hyvrinen, A.-P., Petäjä, T., Vanhanen, J., Sipilä, M., Paasonen, P., Kulmala, M., and Lihavainen, H.: Homogenous nucleation of sulfuric acid and water at close to atmospherically relevant conditions, Atmos. Chem. Phys., 11, 52775287, doi:10.5194/acp-11-5277-2011, 2011.

Chesnokov, E. N. and Krasnoperov, L. N.: Complete thermodynamically consistent kinetic model of particle nucleation and growth: Numerical study of the applicability of the classical theory of homogeneous nucleation, J. Chem. Phys., 126, 144504, 2007.

Courtney, W. G.: Nonsteady-state nucleation, J. Chem. Phys., 36, 2009-2017, 1962.

Dal Maso, M., Kulmala, M., Riipinen, I., Wagner, R., Hussein, T., Aalto, P. P., and Lehtinen, K. E. J.: Formation and growth of fresh atmospheric aerosols: Eight years of aerosol size distribution data from SMEAR II, Hyytiälä, Finland, Boreal Environ. Res., 10, 323-336, 2005.

Dal Maso, M., Hyvärinen, A., Komppula, M., Tunved, P., Kerminen, V.-M., Lihavainen, H., Viisanen, Y., Hansson, H.-C., and Kulmala, M.: Annual and interannual variation in boreal forest aerosol particle number and volume concentration and their connection to particle formation, Tellus, 60B, 495-508, 2008.

Ge, X., Wexler, A. S., and Clegg, S. L.: Atmospheric amines -Part I. A review, Atmos. Environ., 45, 524-546, 2011.

Girshick, S. L. and Chiu, C.-P.: Numerical study of $\mathrm{MgO}$ powder synthesis by thermal plasma, J. Aerosol Sci., 21, 641-650, 1990.

Girshick, S. L., Agarwal, P., and Truhlar, D. G.: Homogeneous nucleation with magic numbers: Aluminum, J. Chem. Phys., 131, 134305, 2009.

Hirsikko, A., Nieminen, T., Gagné, S., Lehtipalo, K., Manninen, H. E., Ehn, M., Hõrrak, U., Kerminen, V.-M., Laakso, L., McMurry, P. H., Mirme, A., Mirme, S., Petäjä, T., Tammet, H., Vakkari, V., Vana, M., and Kulmala, M.: Atmospheric ions and nucleation: a review of observations, Atmos. Chem. Phys., 11, 767-798, doi:10.5194/acp-11-767-2011, 2011.

Hoppel, W. A. and Frick, G. M.: Ion-aerosol attachment coefficients and the steady-state charge distribution on aerosols in a bipolar ion environment, Aerosol Sci. Tech., 5, 1-21, 1986.

Israël, H.: Atmospheric Electricity, vol. I., Israel Program for Sci. Transl. \& NSF, Jerusalem, 1970.

Janson, R., Rosman, K., Karlsson, A., and Hansson, H.-C.: Biogenic emissions and gaseous precursors to forest aerosols, Tellus, 53B, 423-440, 2001.

Kathmann, S. M., Schenter, G. K., and Garrett, B. C.: Multicomponent dynamical nucleation theory and sensitivity analysis, J. Chem. Phys., 120, 9133-9141, 2004.

Kazil, J. and Lovejoy, E. R.: A semi-analytical method for calculating rates of new sulfate aerosol formation from the gas phase, 7 , 3447-3459, 2007.

Koutzenogii, K. P., Levykin, A. I., and Sabelfeld, K. K.: Kinetics of aerosol formation in the free molecule regime in presence of condensable vapor, J. Aerosol Sci., 27, 665-679, 1996.

Kožíšek, Z. and Demo, P.: Influence of initial conditions on homogeneous nucleation kinetics in a closed system, J. Chem. Phys., 123, 144502, 2005.

Kožíšek, Z., Sato, K., Demo, P., and Sveshnikov, A. M.: Homogeneous nucleation of droplets from supersaturated vapor in a closed system, J. Chem. Phys., 120, 6660-6664, 2004.

Kožíšek, Z., Demo, P., and Sveshnikov, A. M.: Size distribution of nuclei in a closed system, J. Chem. Phys., 125, 114504, 2006.

Kulmala, M.: How particles nucleate and grow, Science, 302, 10001001, 2003.

Kulmala, M.: Dynamical atmospheric cluster model, Atmos. Res., 98, 201-206, 2010.

Kulmala, M. and Wagner, P. E.: Mass accommodation and uptake coefficients - a quantitative comparison , J. Aerosol Sci., 32, 833-841, 2001.

Kulmala, M., Dal Maso, M., Mäkelä, J. M., Pirjola, L., Väkevä, M., Aalto, P., Miikkulainen, P., Hämeri, K., and O’Dowd, C. D.: On the formation, growth and composition of nucleation mode particles, Tellus B, 53, 479-490, 2001.

Kulmala, M., Vehkamäki, H., Petäjä, T., Del Maso, M., Lauri, A., Kerminen, V.-M., Birmili, W., and McMurry, P. H.: Formation 
and growth rates of ultrafine atmospheric particles: a review of observations, J. Aerosol Sci., 35, 143-176, 2004.

Kurtén, T.: A comment on Nadytko et al.'s “Amines in the Earth's Atmosphere: A Density Functional Theory Study of the Thermochemistry of Pre-Nucleation Clusters", Entropy, 13, 915-923, 2011.

Kurtén, T., Loukonen, V., Vehkamäki, H., and Kulmala, M.: Amines are likely to enhance neutral and ion-induced sulfuric acid-water nucleation in the atmosphere more effectively than ammonia, Atmos. Chem. Phys., 8, 4095-4103, doi:10.5194/acp8-4095-2008, 2008.

Kurtén, T., Kuang, C., Gómez, P., McMurry, P. H., Vehkamäki, H., Ortega, I., Noppel, M., and Kulmala, M.: The role of cluster energy nonaccommodation in atmospheric sulfuric acid nucleation, J. Chem. Phys., 132, 024304, 2010.

Kurtén, T., Petäjä, T., Smith, J., Ortega, I. K., Sipilä, M., Junninen, H., Ehn, M., Vehkamäki, H., Mauldin, L., Worsnop, D. R., and Kulmala, M.: The effect of $\mathrm{H}_{2} \mathrm{SO}_{4}-$ amine clustering on chemical ionization mass spectrometry (CIMS) measurements of gas-phase sulfuric acid, Atmos. Chem. Phys., 11, 3007-3019, doi:10.5194/acp-11-3007-2011, 2011.

Langevin, P. M.: Une formule fondamentale de théorie cinétique, Ann. Chim. Phys., 8, 245-288, 1905.

Li, Z. H. and Truhlar, D. G.: Cluster and nanoparticle condensation and evaporation reactions. Thermal rate constants and equilibrium constants of $\mathrm{Al}_{m}+\mathrm{Al}_{n-m} \leftrightarrow \mathrm{Al}_{n}$ with $n=2-60$ and $m=1-8$, J. Phys. Chem. C, 112, 11109-11121, 2008.

Li, Z. H., Bhatt, D., Schultz, N. E., Siepmann, J. I., and Truhlar, D. G.: Free energies of formation of metal clusters and nanoparticles from molecular simulations: $\mathrm{Al}_{n}$ with $n=2-60$, J. Phys. Chem. C, 111, 16227-16242, 2007.

Lovejoy, E. R., Curtius, J., and Froyd, K. D.: Atmospheric ioninduced nucleation of sulfuric acid and water, J. Geophys. Res., 109, D08204, (doi:10.1029/2003JD004460), 2004.

Manninen, H. E., Nieminen, T., Riipinen, I., Yli-Juuti, T., Gagné, S., Asmi, E., Aalto, P. P., Petäjä, T., Kerminen, V.-M., and Kulmala, M.: Charged and total particle formation and growth rates during EUCAARI 2007 campaign in Hyytiälä, Atmos. Chem. Phys., 9, 4077-4089, doi:10.5194/acp-9-4077-2009, 2009.

McGraw, R.: Two-dimensional kinetics of binary nucleation in sulfuric acid-water mixtures, J. Chem. Phys., 102, 2098-2108, 1995.

Nadykto, A. B., Yu, F., Jakovleva, M. V., Herb, J., and Xu, Y.: Amines in the Earth's atmosphere: a density functional theory study of the thermochemistry of pre-nucleation clusters, Entropy, 13, 554-569, 2011.

Nishioka, K. and Fujita, K.: Transient nucleation in binary vapor of water and sulfuric acid, J. Chem. Phys., 100, 532-540, 1994.

Ortega, I. K., Kupiainen, O., Kurtén, T., Olenius, T., Wilkman, O., McGrath, M. J., Loukonen, V., and Vehkamäki, H.: From quantum chemical formation free energies to evaporation rates, Atmos. Chem. Phys., 12, 225-235, doi:10.5194/acp-12-225-2012, 2012.
Petäjä, T., Mauldin III, R. L., I., Kosciuch, E., McGrath, J., Nieminen, T., Paasonen, P., Boy, M., Adamov, A., Kotiaho, T., and Kulmala, M.: Sulfuric acid and $\mathrm{OH}$ concentrations in a boreal forest site, Atmos. Chem. Phys., 9, 7435-7448, doi:10.5194/acp9-7435-2009, 2009.

Petäjä, T., Sipilä, M., Paasonen, P., Nieminen, T., Kurtén, T., Ortega, I. K., Stratmann, F., Vehkamäki, H., Berndt, T., and Kulmala, M.: Experimental observation of strongly bound dimers of sulfuric acid: Application to nucleation in the atmosphere, Phys. Rev. Lett., 106, 228302, 2011.

Pöschl, U.: Atmospheric aerosols: Composition, transformation, climate and health effects, Angew. Chem., Int. Ed., 44, 75207540, 2005.

Rao, N. P. and McMurry, P. H.: Nucleation and growth of aerosol in chemically reacting systems, Aerosol Sci. Tech., 11, 120-132, 1989.

Shampine, L. F. and Reichelt, M. W.: The MATLAB ODE suite, SIAM J. Sci. Comput., 18, 1-22, 1997.

Sipilä, M., Lehtipalo, K., Attoui, M., Neitola, K., Petäjä, T., Aalto, P. P., O'Dowd, C. D., and Kulmala, M.: Laboratory verification of PH-CPC's ability to monitor atmospheric sub-3 nm clusters, Aerosol Sci. Tech., 43, 126-135, 2009.

Sipilä, M., Berndt, T., Petäjä, T., Brus, D., Vanhanen, J., Stratmann, F., Patokoski, J., Mauldin III, R. L., Hyvärinen, A.-P., Lihavainen, H., and Kulmala, M.: The role of sulfuric acid in atmospheric nucleation, Science, 327, 1243-1246, 2010.

Vanhanen, J., Mikkilä, J., Lehtipalo, K., Sipilä, M., Manninen, H. E., Siivola, E., Petäjä, T., and Kulmala, M.: Particle size magnifier for nano-CN detection, Aerosol Sci. Tech., 45, 533-542, 2011.

Vehkamäki, H., Paatero, P., Kulmala, M., and Laaksonen, A.: Erratum:Binary Nucleation Kinetics: A Matrix Method, (J. Chem. Phys., 101, 9997-10002, 1994), J. Chem. Phys., 106, 2987, 1997.

Vehkamäki, H., McGrath, M. J., Kurtén, T., Julin, J., Lehtinen, K. E. J., and Kulmala, M.: Rethinking the application of the first nucleation theorem to particle formation, J. Chem. Phys., in press, 2012.

Wyslouzil, B. E. and Wilemski, G.: Binary nucleation kinetics. II. Numerical solution of the birth-death equations, J. Chem. Phys., 103, 1137-1151, 1995.

Yu, F.: Quasi-unary homogeneous nucleation of $\mathrm{H}_{2} \mathrm{SO}_{4}-\mathrm{H}_{2} \mathrm{O}$, J. Chem. Phys., 122, 074501, 2005.

Yu, F.: Effect of ammonia on new particle formation: A kinetic $\mathrm{H}_{2} \mathrm{SO}_{4}-\mathrm{H}_{2} \mathrm{O}-\mathrm{NH}_{3}$ nucleation model constrained by laboratory measurements, J. Geophys. Res., 111, D01204, doi:10.1029/2005JD005968, 2006. 